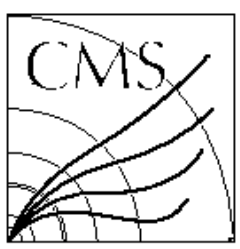

The Compact Muon Solenoid Experiment

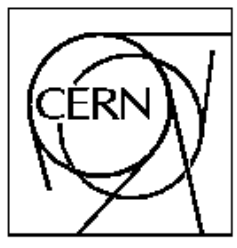

June 9, 2003

\title{
Proton irradiation of analogue opto-hybrid circuits for the CMS tracker
}

\author{
M.T. Brunetti ${ }^{\mathrm{a}}$, B. Checcucci ${ }^{\mathrm{a}}$, V. Postolache ${ }^{\mathrm{a}}$, D. Ricci ${ }^{\mathrm{a}}$ \\ T. Bauer ${ }^{\mathrm{b}}$, M. Friedl ${ }^{\mathrm{b}}$ and M. Pernicka ${ }^{\mathrm{b}}$ \\ ${ }^{a}$ INFN and University of Perugia, Via A. Pascoli 1, I-06123 Perugia \\ ${ }^{b}$ HEPHY, Nikolsdorfergasse 18, A-1050 Wien
}

\begin{abstract}
Four analogue opto-hybrid circuits to be used in the front-end electronics of the CMS (Compact Muon Solenoid) tracker were irradiated with $26 \mathrm{MeV}$ protons to fluences of $0.5 \cdot 10^{14} \mathrm{p} / \mathrm{cm}^{2}$ and $0.8 \cdot 10^{14} \mathrm{p} / \mathrm{cm}^{2}$ in the secondary beam of the cyclotron at the Forschungszentrum (FZK) in Karlsruhe (Germany). The measurements done during the irradiation are reported and discussed and the circuit performance, tested before and after the irradiation, is compared.
\end{abstract}




\section{Introduction}

The CMS silicon tracker [1], with its capability of collecting a huge amount of data from high energy proton-proton interactions in the LHC tunnel, is an outstanding example of the frontiers achieved in the fields of physics and technology.

About 10 million channels will be involved in the trajectory reconstruction and this has led to the development of front-end electronics which are fast, compact and very resistant to the harsh radiation environment. The calculated energy integrated fluence for fast hadrons in 10 years is $1.6 \cdot 10^{14} \mathrm{~cm}^{-2}$ in the inner tracker layer and $0.35 \cdot 10^{14} \mathrm{~cm}^{-2}$ in the most outer layer [1].

Optical links [2,3], typically $60 \mathrm{~m}$ long, will allow the transfer of data at a frequency of $40 \mathrm{MHz}$ from the detector to the control room, where analogue pulses are digitised and processed at the Front-End Driver (FED) [4]. Two types of optical links will be used in the CMS tracker, a bi-directional digital control link and an analogue readout link.

The conversion of analogue electrical signals into the corresponding optical ones is done by means of an analogue opto-hybrid (AOH) circuit. Several versions of it were developed and tested by the CMS collaboration in Perugia, Vienna and at CERN [5,6]. The specific geometric requirements for the various parts of the tracker, i.e. inner barrel and inner disks (TIB/TID), outer barrel (TOB) and end caps (TEC) led to three layouts of the $\mathrm{AOH}$ circuit. The total number of AOHs to be built and tested for the CMS tracker amounts to about 17000 . Figure 1 shows the scheme of the analogue part of the optical link. The pulses induced by charged particles passing through the silicon detector are sampled and multiplexed by APV chips [7] and MUX. The resulting differential input voltage is converted by the $\mathrm{AOH}$ into an analogue optical signal and is transmitted via optical fibres to the back-end electronics. Each fibre will carry the multiplexed signals of 256 microstrips.

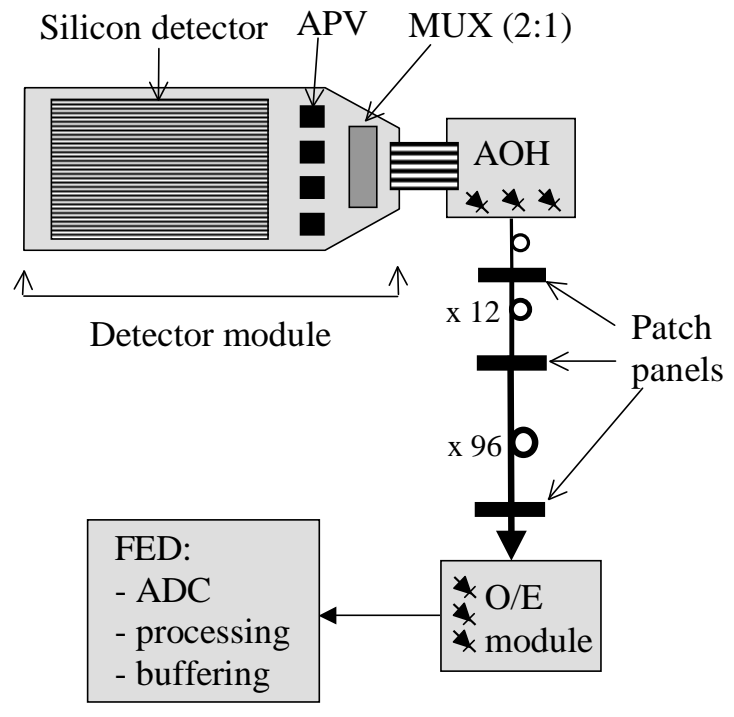

Figure 1: Scheme of the analogue part of the CMS tracker optical readout link.

Patch panels group together the fibres into optical cables that arrive at the back end electronics in the control room. Here optical signals are re-converted into electrical and are processed.

\section{The analogue opto-hybrid circuit}

The analogue opto-hybrid circuit for the CMS silicon tracker consists of a FR4 (vetronite) substrate with dimensions of $30 \times 22 \times 0.5 \mathrm{~mm}^{2}$ for the TIB/TID and slightly larger, $30 \times 23 \times 0.5 \mathrm{~mm}^{2}$, for the TOB and TEC.

All three AOH types are equipped with one linear laser driver (LLD) chip [8] and 2 or 3 laser diodes. The LLD is programmable via an $\mathrm{I}^{2} \mathrm{C}$ interface and can be set to bias the laser diodes in their linear operational region. Four increasing gains $(5,7.5,10$ and $12.5 \mathrm{mS})$ of the differential input signals are pre-settable through the LLD in order to compensate for threshold variations due to radiation damage. 
The laser diodes are commercially available InGaAsP edge-emitting coupled to single mode optical fibers operating at a wavelength of $1310 \mathrm{~nm}$. They are glued to the substrate with a thermally conductive resin which is resistant to radiation and are ultrasonically bonded. A version of the TOB AOH with laser diodes soldered to the substrate has also been developed, but will not be used in the final system.

Figure 2 shows the pictures of the AOHs irradiated in Karlsruhe, where (a) shows the TIB/TID AOH front and back side, (b) the TOB AOH with laser diodes bonded on the substrate and (c) the TOB AOH with soldered laser diodes.

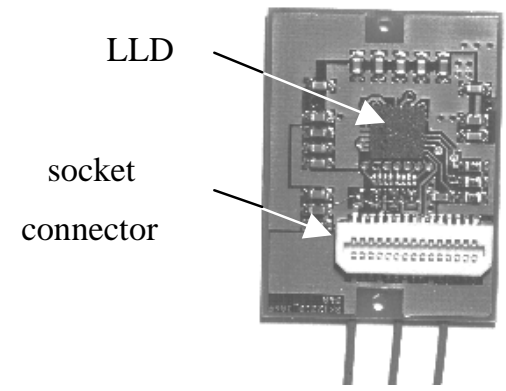

header

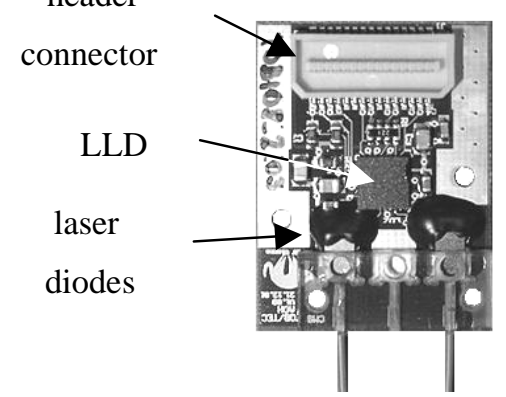

(b)

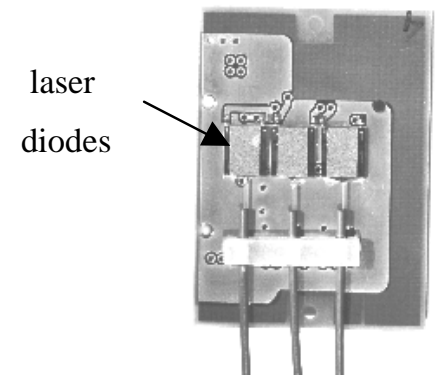

(a)

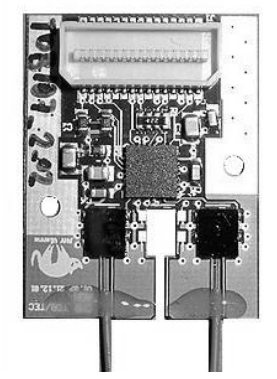

(c)

Figure 2. (a) Front side and back side of the TIB/TID analogue opto-hybrid. (b) TOB analogue opto-hybrid in the bonded version. (c) TOB analogue opto-hybrid in the soldered version.

The link to the detector module is done through a 30-pin NAIS connector, socket type for the TIB/TID and TEC and header type for the TOB. Due to mechanical constraints, in the TIB/TID AOH the LLD and the connector are on the opposite side of the substrate with respect to the laser diodes, while they are on the same side for the TOB and TEC versions. In order to protect the bonds during assembly and handling, wires are hard covered with a plastic (ABS) stamp (not present in fig. 2).

\section{Proton irradiation}

The irradiation of the analogue opto-hybrid is the final step in the qualification of this circuit for resistance to radiation damage.

Previous qualification was dedicated only to components of the $\mathrm{AOH}$, i.e. laser diodes, fibres and connectors [9,10], LLD [8], substrate, passive electrical components and glues [11]. The results obtained already gave a good level of confidence on the $\mathrm{AOH}$ radiation hardness. Nevertheless, the response to radiation had also to be proven once assembled with all its parts.

Four AOHs, two TIB/TID and two TOB, were irradiated in a secondary beam of the KIZ isochrone cyclotron at the Forschungzentrum in Karlsruhe. The irradiation source is a proton beam of $26 \mathrm{MeV}$ with a current adjustable from $400 \mathrm{nA}$ to $2 \mu \mathrm{A}$. Figure 3 shows the beam area. 


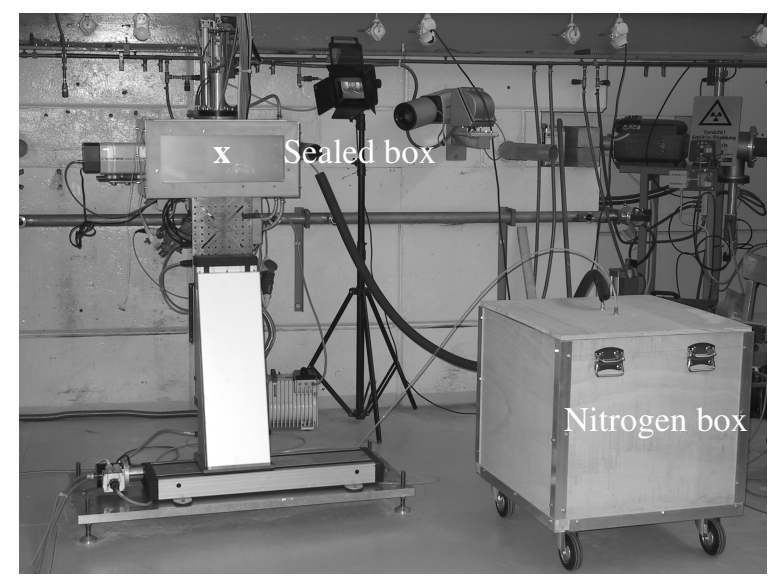

Figure 3. Picture of the cyclotron beam area with the scanning system facility.

A scanning system, placed on a rail, allows uniform irradiation of the target. The circuits are contained inside the sealed box placed in front of the beam (represented by the white $\mathrm{X}$ in fig. 3 ). The box to the right contains the nitrogen used to cool the target.

The electrical setup used in the AOH's irradiation was built to drive the $\mathrm{AOH}$ circuit without the detector module, like in the final readout chain shown in fig. 1. It is represented by the scheme in figure 4.

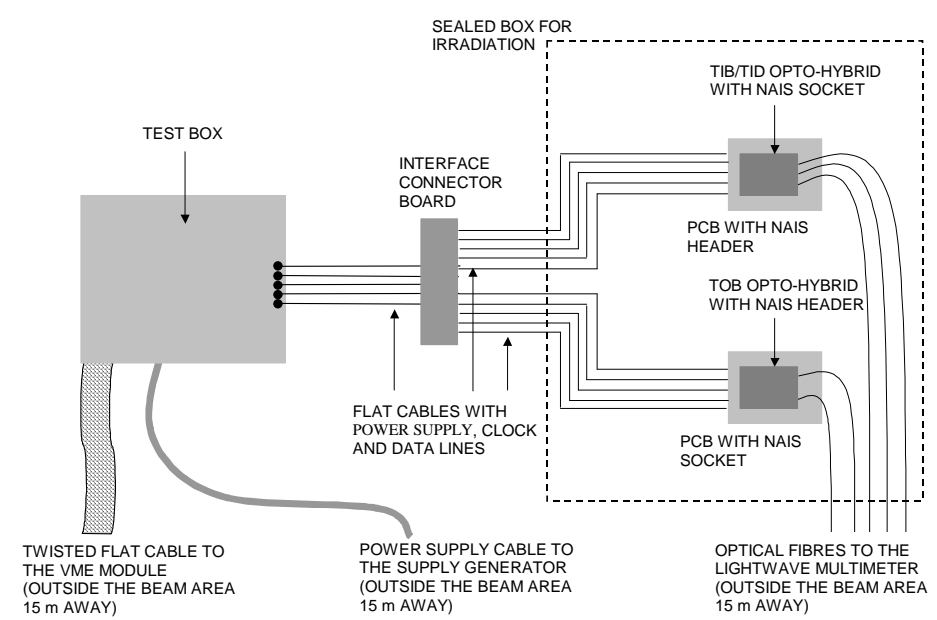

Figure 4. Scheme of the setup in the cyclotron beam area used to irradiate the 4 AOHs.

The AOHs are plugged onto two PCBs inside the sealed box, represented by the dotted rectangle in the right of the figure. They are powered and biased through the five line flat cables $\left(\mathrm{V}_{\mathrm{DD}}, \mathrm{V}_{\mathrm{SS}}, \mathrm{V}_{125}\right.$, clock and data) arriving from the front-end electronics (gray test box on the left). An interface connector board (centre of the figure) splits the signals from the test box to feed both AOHs under test. The optical fibres from the AOHs run outside the beam area to an optical power-meter (Agilent 8163A lightwave multimeter). The twisted flat cable and the power supply cable go, respectively, to a VME module and power supply generator located in the control room.

Two irradiation runs were done, with $2 \mathrm{AOHs}$ at each time. Several beam stops were requested during the test, in order to monitor the rate of degradation after each irradiation step. In the first irradiation run the beam current was initially set to $400 \mathrm{nA}$ and then rose up to $1 \mu \mathrm{A}$ after 10 steps, reaching a total fluence of $0.54 \cdot 10^{14}$ $\mathrm{p} / \mathrm{cm}^{2}$. The second irradiation was performed in $10 \mathrm{steps}$, each about $1 \mathrm{~min}$ long, with a beam current of $1 \mu \mathrm{A}$ and a final fluence of $0.76 \cdot 10^{14} \mathrm{p} / \mathrm{cm}^{2}$. Both fluences are calculated with the beam current and are affected by a systematic error of $15 \%$. The temperature inside the sealed box was measured by a probe placed near the AOHs and varied between -8 and $-2{ }^{\circ} \mathrm{C}$ during the first irradiation run and between -18 and $-14{ }^{\circ} \mathrm{C}$ during the second. All the results reported in this paper refer to the two AOHs (one TIB/TID and the soldered TOB) irradiated at higher fluence. 


\section{Monitoring during the irradiation}

The signals carried from two optical fibres (one for each $\mathrm{AOH}$ ) were measured during the irradiation test by the Agilent 8163 A lightwave multimeter.

The measurement of the output light power as a function of time is shown in figures $5 \mathrm{a}$ and $5 \mathrm{~b}$ for some increasing steps of the irradiation. The temperature inside the cooled box is reported together with the increasing fluence value. Before the beam was switched on, the laser diode was biased with a current of $45 \mathrm{~mA}$, well above its threshold current that is usually around $5 \mathrm{~mA}$. The initial decrease of the output light power in figs. $5 \mathrm{a}$ and $5 \mathrm{~b}$ is related to the self-heating of the laser diode. Once the output power is settled, after about $60 \mathrm{~s}$, the $\mathrm{AOH}$ is ready to be irradiated. When the beam crosses the laser diode under test, the emitted power suddenly decreases until the beam stop. A partial recovery of the initial light power was observed after some tens of second. The maximum variation of the initial output light power during the irradiation amounts to about $100 \mu \mathrm{W}$ for the TIB/TID AOH and about $200 \mu \mathrm{W}$ for the TOB AOH. The relationship between this variation and the irradiation is not so evident, since there is no clear correlation in these results between the expected decrease in laser diode efficiency with increasing fluence. This is instead more evident in the second type of monitoring described below.

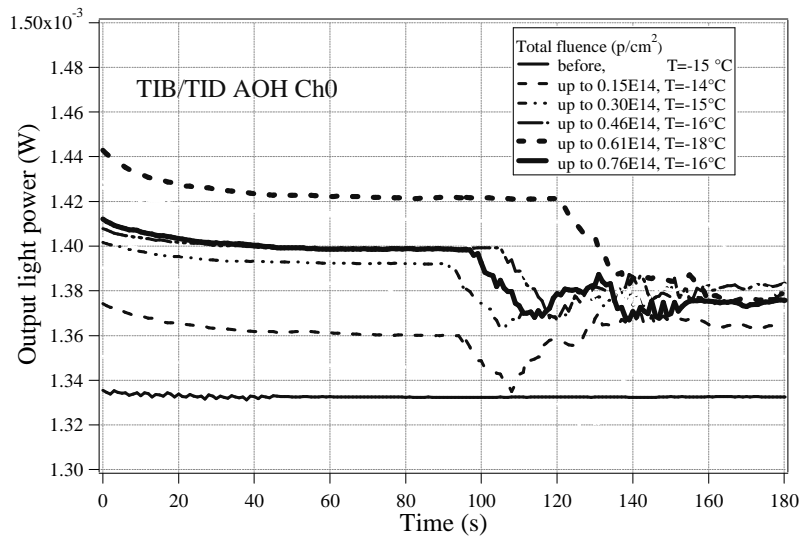

(a)

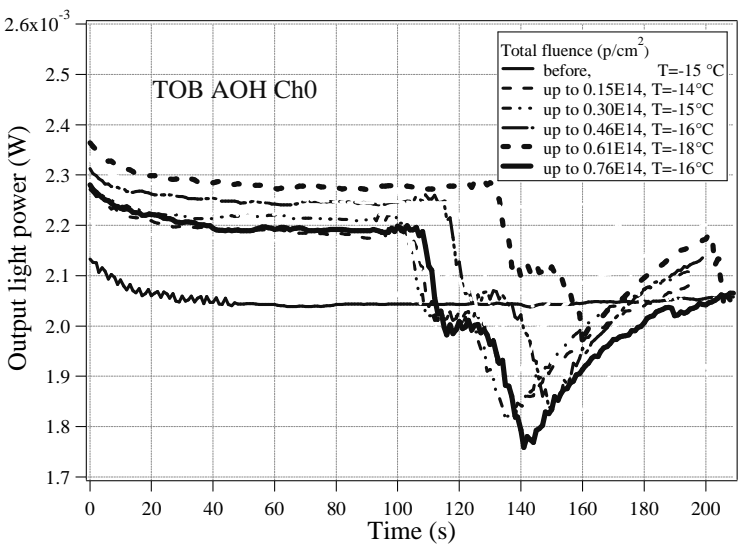

(b)

Figure 5. Output light power during the irradiation as a function of time for the TIB/TID (a) and TOB (b) AOH. The temperature measured by the probe inside the sealed box is also reported.

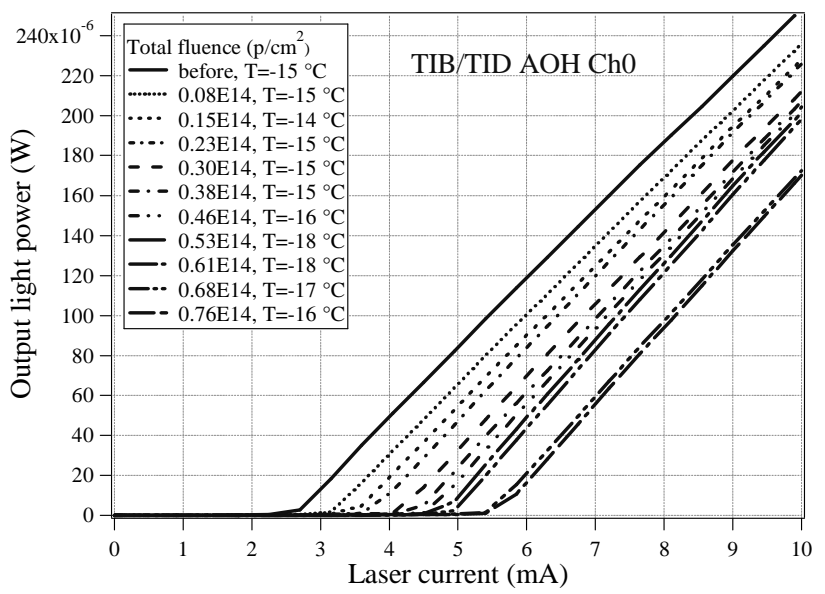

(a)

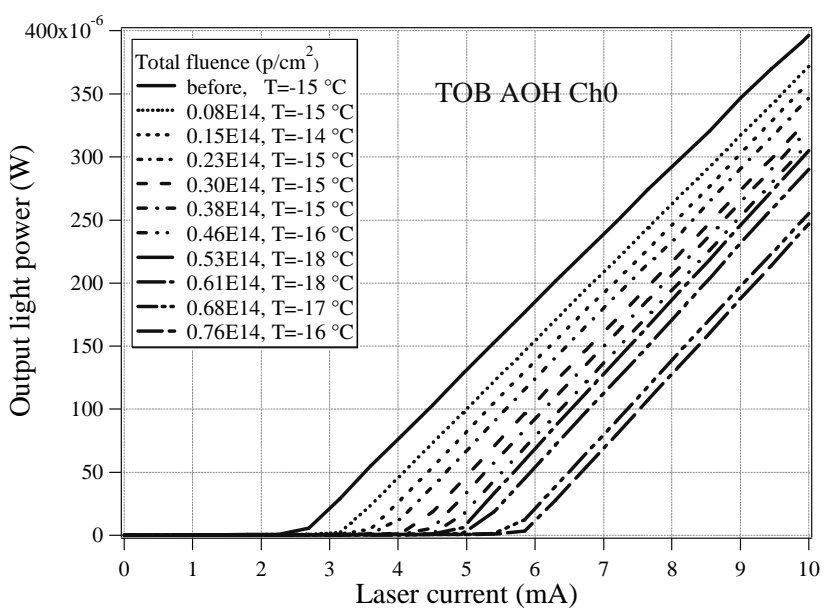

(b)

Figure 6. Output light power as a function of bias current after each step of irradiation for the TIB/TID (a) and TOB (b) AOH. 
The measurement of the output light power as a function of the bias current of the same two laser diodes (channel 0 of each $\mathrm{AOH}$ ) was done soon after each irradiation step and is shown in figure 6 . The diodes were biased with an increasing current from 0 to $54 \mathrm{~mA}$ in steps of $0.45 \mathrm{~mA}$, that corresponds to one unit of the LLD register. In particular, the data in fig. 6 were plotted in the range 0-10 mA in order to show the laser threshold shift of about $3 \mathrm{~mA}$ caused by the irradiation. The temperature inside the cooled box was measured by a local probe, placed near the AOHs, and is reported together with the increasing fluence value. The dependence of the laser threshold on the temperature for non irradiated $\mathrm{AOHs}$ was measured in the range $-15{ }^{\circ} \mathrm{C}$ to $25{ }^{\circ} \mathrm{C}$. According to these measurements, the temperature variation reported in fig. 6 contributes to less than $10 \%$ of the total current shift.

\section{Characterization before and after the irradiation}

The monitoring of the AOH's output light during the irradiation is useful to check the immediate effect of the irradiation in the circuit behaviour. More specific tests were performed after 3 months, when the activity of the irradiated AOHs allowed a safe handling. These are the measurement, at room temperature (between $25^{\circ} \mathrm{C}$ and $28{ }^{\circ} \mathrm{C}$ ) of some static and dynamic electrical parameters, i.e. gain, integral linearity deviation, RMS noise, bandwidth and crosstalk. The setup scheme for the $\mathrm{AOH}$ characterization before and after the irradiation is shown in figure 7.

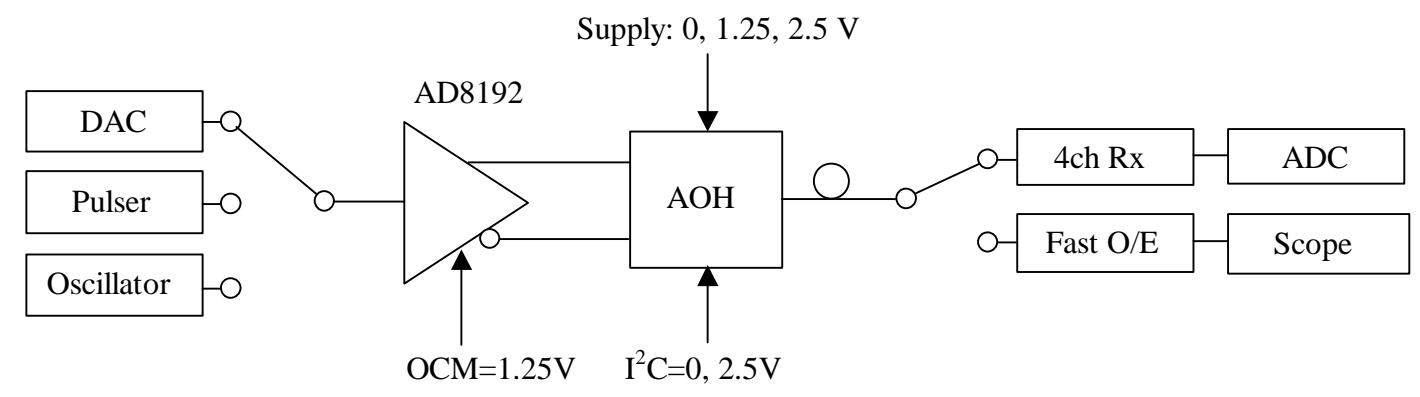

Figure 7. Scheme of the setup used for the AOH's electrical characterization before and after the irradiation.

The AOHs are biased slightly above the laser diode threshold, in their linear operational range.

The gain is the slope of the linear fit of the output voltage as a function of the input voltage in the range $300 \div 300 \mathrm{mV}$. The input pulse is generated by the DAC and is converted into a differential signal by the Analog Devices AD8192 amplifier. The optical output signal is transferred through the optical fibres to a 4-channel receiver module where it is converted back to an electrical output and sent to the ADC. Table I states the gain values measured on channels 0 and 1 of the TIB/TID AOH (a), and channels 0 and 2 of the TOB AOH (b), before (upper value) and after (lower value) the irradiation. The measurements show a general decrease of the gain, of $14 \%$ on average (with a spread between 0 and 30\%) due to the radiation damage, which causes a lack of efficiency in laser diodes. The four columns correspond to the four LLD pre-settable gains (5, 7.5, 10 and 12.5 $\mathrm{mS})$.

The integral linearity deviation (or integral non linearity, INL) is also calculated by the linear fit of the output voltage of the optical link as a function of the differential input voltage in the interval $-300 \div 300 \mathrm{mV}$ (operational voltage range). The measurements before (a) and after (b) the irradiation are reported in figure 8 for the TIB/TID and TOB AOHs for a LLD gain setting of $5 \mathrm{mS}$. The horizontal solid lines in fig. 8(a) and 8(b) correspond to the maximum value of $1.5 \%$ for INL in the operational voltage range, as requested by the CMS tracker optical link specifications [12]. Both channels of the two AOHs satisfy this constraint, before and after the irradiation.

The output voltage RMS noise is measured by a high bandwidth ADC for differential input voltage levels varying from -300 and $300 \mathrm{mV}$. The results are reported in figure 9 before (a) and after (b) the irradiation. Some increase in the noise after the irradiation was observed in all channels except for channel 2 of the TOB AOH, where even some decrease is present. The horizontal solid lines are again the limits for the average noise allowed by the specifications for the CMS tracker optical link [12]. 
Table I: Gain values measured on the two channels of each AOH before (upper) and after (lower) the irradiation at the fluence of $0.76 \cdot 10^{14} \mathrm{p} / \mathrm{cm}^{2}$.

TIB/TID AOH

\begin{tabular}{|c|c|c|c|c|}
\hline & \multicolumn{4}{|c|}{ Gain $(\mu \mathrm{W} / \mathrm{mV})$} \\
\hline Ch. & $\begin{array}{c}\text { LD gain } \\
5 \mathrm{mS}\end{array}$ & $\begin{array}{c}\text { LD gain } \\
7.5 \mathrm{mS}\end{array}$ & $\begin{array}{c}\text { LD gain } \\
10 \mathrm{mS}\end{array}$ & $\begin{array}{l}\text { LD gain } \\
12.5 \mathrm{mS}\end{array}$ \\
\hline \multirow{2}{*}{0} & 0.18 & 0.27 & 0.36 & 0.45 \\
\hline & 0.17 & 0.25 & 0.34 & 0.35 \\
\hline \multirow{2}{*}{1} & 0.18 & 0.27 & 0.37 & 0.42 \\
\hline & 0.13 & 0.20 & 0.26 & 0.32 \\
\hline
\end{tabular}

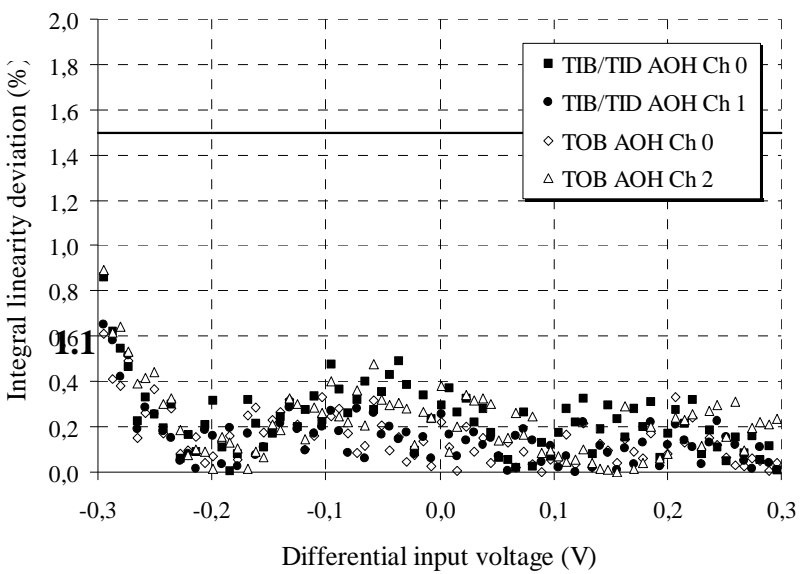

(a)
TOB AOH

\begin{tabular}{|c|c|c|c|c|}
\hline \multicolumn{4}{|c|}{ Gain $(\mu \mathrm{W} / \mathrm{mV})$} \\
\hline \multirow{2}{*}{ Ch. } & LD gain & LD gain & LD gain & LD gain \\
\hline \multirow{3}{*}{0} & $5 \mathrm{mS}$ & $7.5 \mathrm{mS}$ & $10 \mathrm{mS}$ & $12.5 \mathrm{mS}$ \\
& 0.14 & 0.20 & 0.28 & 0.35 \\
& 0.14 & 0.21 & 0.28 & 0.35 \\
\hline 2 & 0.20 & 0.30 & 0.40 & 0.50 \\
& 0.16 & 0.24 & 0.32 & 0.39 \\
\hline
\end{tabular}

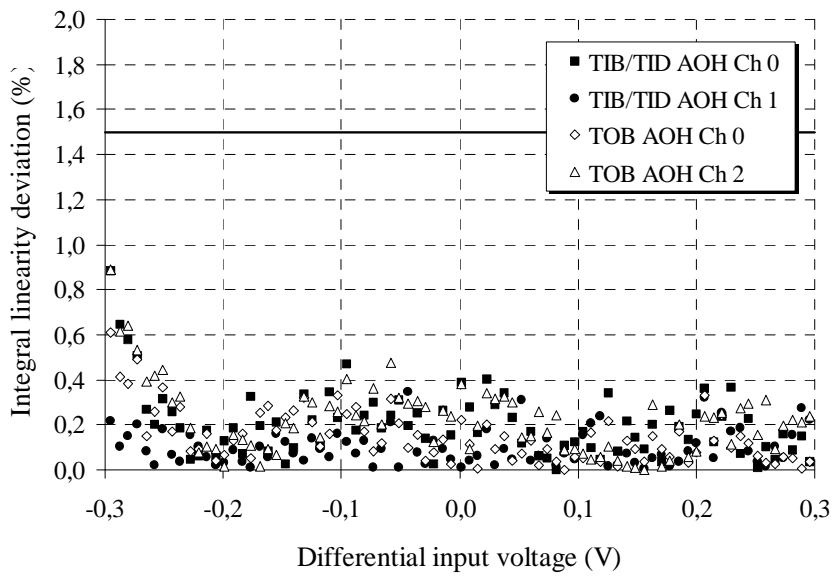

(b)

Figure 8. Integral linearity deviation on two channels of the TIB/TID and TOB AOHs before (a) and after (b) the irradiation.

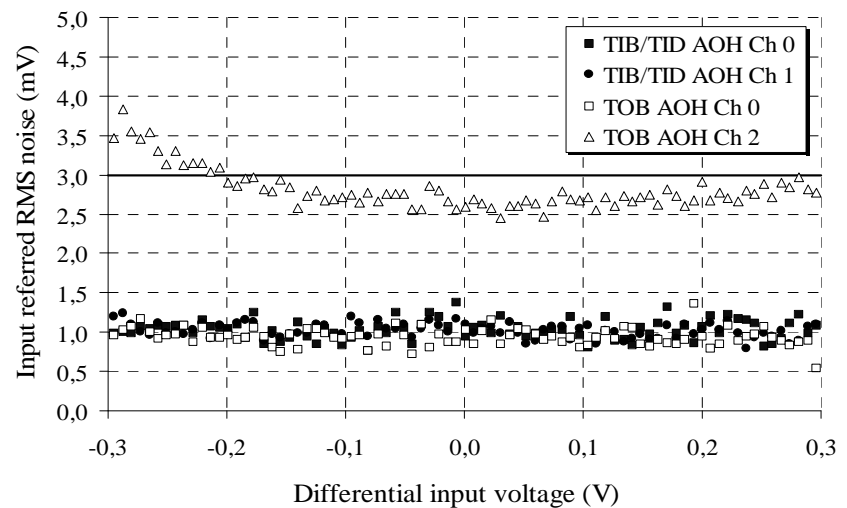

(a)

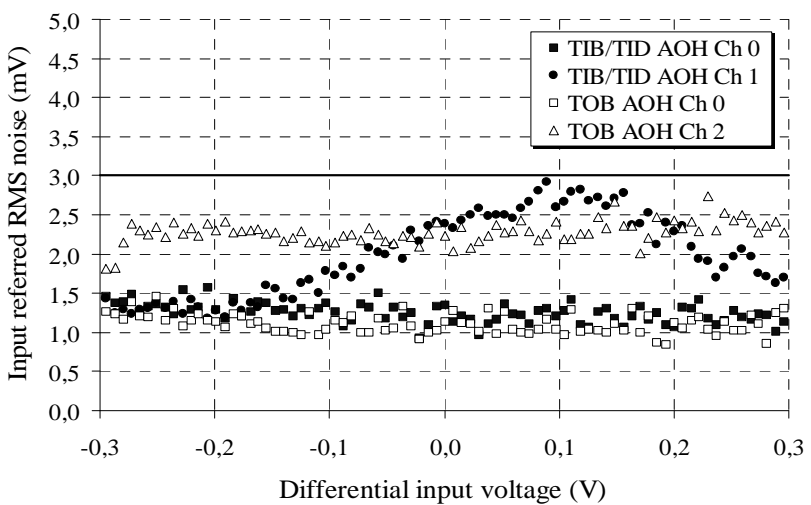

(b)

Figure 9. RMS noise on two channels of the TIB/TID and TOB AOHs before (a) and after (b) the irradiation.

The important result is that both RMS noise and INL are only weakly affected by the irradiation.

The bandwidth of the two AOH circuits was measured by generating sine waves at increasing frequencies with a Rhode \& Schwartz SMX RF oscillator (range 0.1 to $1000 \mathrm{MHz}$ ) (see fig. 7). The optical output is converted into electrical by the high frequency photodiode (Terahertz Technologies TIA-950) and read on the digital scope (Tektronix TDS 3054, bandwidth $500 \mathrm{MHz}$ ). The ratio between the amplitude of the output signal 
and that of the input gives a measurement of the bandwidth at $-3 \mathrm{~dB}$. A comparison of the results obtained before and after the irradiation is reported in figure 10 . The bandwidth at $-3 \mathrm{~dB}$ (this value is indicated by the horizontal solid line) measured on the two channels of each AOH before and after the irradiation is about 100 $\mathrm{MHz}$, above the minimum of $90 \mathrm{MHz}$ requested by the CMS tracker optical link specifications [12]. The measured values are in fact limited by the bandwidth of the AD8192 amplifier rather than the optical link itself.

The crosstalk between the $\mathrm{AOH}$ channels was measured by injecting a fast input signal from the pulse generator (Agilent 81110A/81112A, rise time $0.8 \mathrm{~ns}$ ) (see fig. 7) on one channel and measuring the corresponding output on the other after $20 \mathrm{~ns}$ with the scope. Figure 11 shows the crosstalk on channel 0 of the TIB/TID AOH before and after the irradiation. The limits, represented in the figure with the dashed lines, correspond to the maximum value of the crosstalk allowed by the CMS tracker specifications for the optical link of $-54 \mathrm{~dB}[12]$.

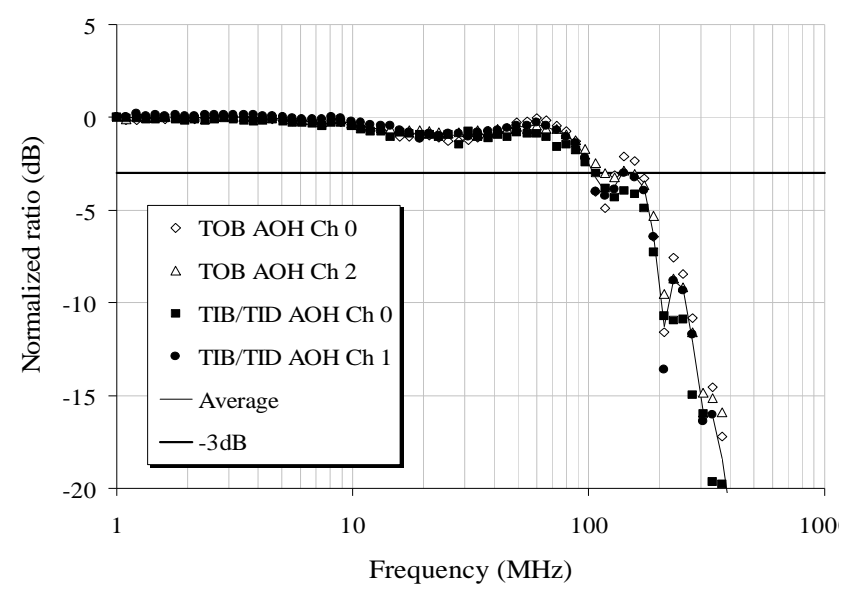

(a)

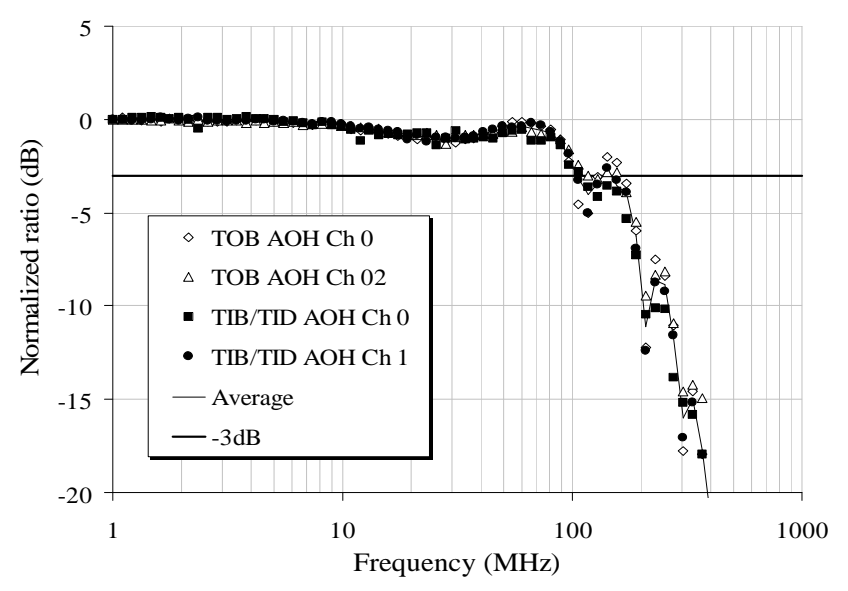

(b)

Figure 10. Bandwidth of two channels of the TIB/TID and TOB AOHs before (a) and after (b) the irradiation.

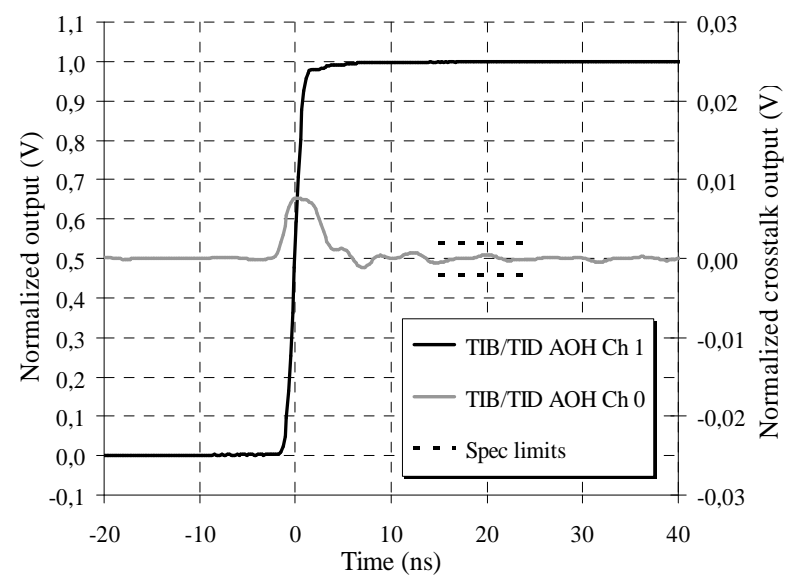

(a)

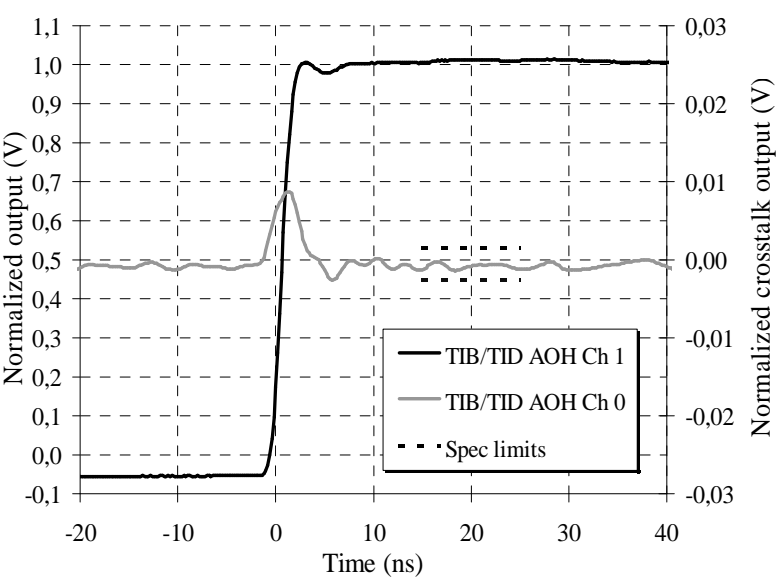

(b)

Figure 11. Right axis: crosstalk measured on channel 0 of the TIB/TID AOH before (a) and after (b) the irradiation. Left axis: normalized output voltage on channel 1.

\section{Conclusions}

The irradiation of analogue opto-hybrid circuits with a proton beam of $26 \mathrm{MeV}$ up to a fluence of $0.8 \cdot 10^{14}$ $\mathrm{p} / \mathrm{cm}^{2}$ has shown an increase of the laser diode threshold current of about $3 \mathrm{~mA}$. Some decrease of the gain of about $14 \%$ on average was measured. The small effect on electrical parameters, like linearity, noise, crosstalk 
and bandwidth demonstrates the radiation tolerance of such electronic devices for proper functionality during the CMS lifetime. These low levels of damage are also consistent with earlier test results and can be easily compensated by the LLD.

\section{Acknowledgements}

We thank A. Dierlamm and F. Hartmann of the Forschungzentrum of Karlsruhe for their collaboration and K. Gill from CERN for useful discussions about the irradiation of optical components. We are grateful to F. Casinini for his support to the test.

\section{References}

[1] "The Tracker Project - Technical Design Report", CERN/LHCC 98-6 (15 April 1998) and "Addendum to the CMS Tracker TDR by the CMS Collaboration”, CERN/LHCC 2000-016 (21 February 2000).

[2] Complete informations available at the URL: http://cern.ch/cms-opto

[3] "Optical readout and control systems for the CMS Tracker". J. Troska et al. To appear on Proc. of the Nuclear Science Symposium, Norfolk, VA, November 2002.

[4] "A PMC based ADC card for CMS Tracker Readout". S. A. Baird et al. Ref.: IEEE Real-Time '99 Conference, Santa Fe, USA.

[5] "Design and performances of a circuit for the analogue optical transmission in the CMS Inner Tracker". M. T. Brunetti et al. Proc. of the $7^{\text {th }}$ LEB Workshop, Stockholm, pp.165-168 (2001).

[6] "Prototype analogue optohybrids for the CMS Outer Barrel and Endcap Tracker". J. Troska et al. Proc. of the $7^{\text {th }}$ LEB Workshop, Stockholm, pp.174-178 (2001).

[7] "The APV25 deep submicron readout chip for CMS detectors". L. L. Jones et al. Proc. of the $5^{\text {th }} L E B$ Workshop, Snowmass-Colorado (1999).

[8] "A radiation tolerant laser driver array for optical transmission in the LHC experiment". G. Cervelli et al. Proc. of the $7^{\text {th }}$ LEB Workshop, Stockholm, pp.155-159 (2001).

[9] K. Gill et al, Photonics for Space and Radiation Environments VIII, Proc. SPIE Vol. 4823, pp. 19-33 (2002).

[10] "Quality assurance programme for the environmental testing of CMS Tracker optical links". K. Gill et al. Proc. of the $7^{\text {th }}$ LEB Workshop, Stockholm, pp.160-164 (2001).

[11] Private communications available at the URLs: http://cms.pg.infn.it/opto/characterization.html and http://aoh.hephy.at/aoh_components_talk_dec2001.pdf.

[12] “CMS Tracker Optical Readout Link Specifications. Part 2: Analogue Opto-hybrid”. Version 3.2, 30 April 2001 CERN EP/CME. Available at the URL: http://edms.cern.ch/document/312573/3.2 